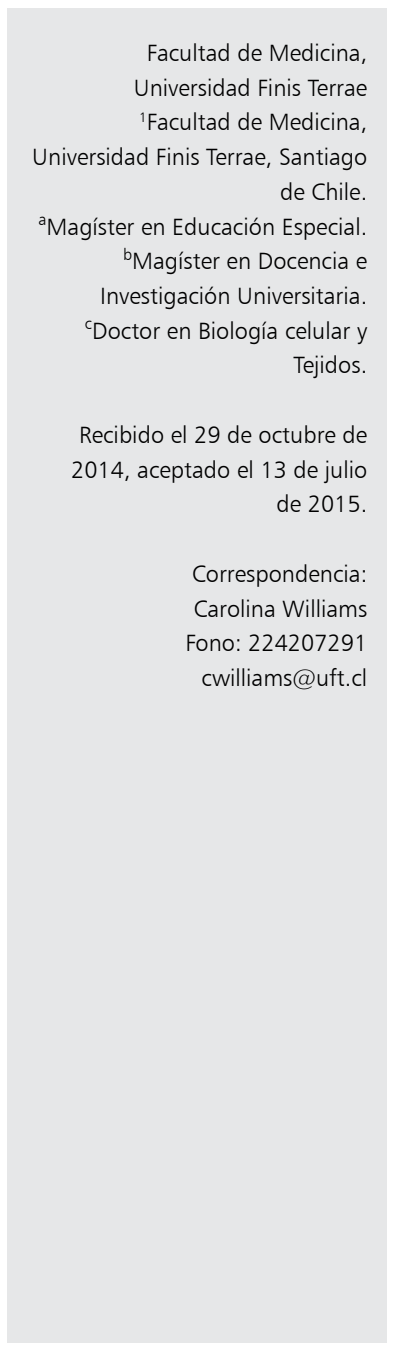

\section{Evaluación de un programa de perfeccionamiento docente implementado en la Facultad de Medicina de la Universidad Finis Terrae}

\author{
LUCÍA SANTELICES ${ }^{1, \mathrm{a}}$, CAROLINA WILLIAMS ${ }^{1, \mathrm{~b}}$, \\ MAURICIO SOTO ${ }^{1, \mathrm{c}}$, ALBERTO DOUGNAC ${ }^{1}$
}

\section{Evaluation of a teaching training program implemented in a faculty of medicine}

Background: Since 2010, the Faculty of Medicine at Finis Terrae University implemented a training program aiming to train health professionals in effective educational practices. Aim: To evaluate the effect of training in teachers who are in charge of planning courses, conducting classes and learning assessment. Material and Methods: Quality of planning, lecture performance and academic performance of students were evaluated in 55 teachers prior and after attending the training course on teaching methodologies and in 47 teachers not attending the course. Results: The percentage of trained teachers complying with the aforementioned indicators was significantly higher than those without training $(p<0.01)$. There were significant differences in favor of the group of teachers who attended and passed the Diploma. Trained teachers had significantly higher students' approval rate. (Odds ratio 4.5, $p<0.01$ ). Conclusions: The teaching Diploma in Health Sciences improved the planning, teaching and academic performance of teachers.

(Rev Med Chile 2015; 143: 1152-1161)

(Key words: Academic training; Education, medical; Planning, techniques; Teaching)

\section{L} a sociedad actual plantea la necesidad de contar con nuevos perfiles de profesionales, capaces de desenvolverse adecuadamente en un mundo globalizado. Frente a este desafío, las instituciones de Educación Superior deben responder con un nuevo proyecto educativo que debe hacerse cargo de nuevos requerimientos no alcanzables con las prácticas docentes tradicionales ${ }^{1}$.

Tradicionalmente los profesionales de la salud que se dedican a la docencia, no poseen una formación que les permita implementar de manera adecuada los nuevos desafíos. Habitualmente la enseñanza tradicional se realiza en forma intuitiva, sin un enfoque educacional. No se facilita el razonamiento clínico, la formación de redes de conocimiento, ni su transferencia al campo de aplicación en el cual deben demostrar sus competencias. Existe poca evidencia acerca del constructo desempeño docente y de cuáles serían las formas correctas de conducir el proceso de enseñanza-aprendizaje para dar cumplimiento a estas nuevas demandas ${ }^{2-4}$.

En educación médica muchos estudios han intentado abordar lo anterior para resolver estos desafíos. No obstante, la información entregada no es suficiente para sacar conclusiones y mejorar los desempeños.

Surge entonces la necesidad de implementar programas de perfeccionamiento en el ámbito de la educación en salud, para satisfacer las demandas 
de la sociedad y mejorar la efectividad del proceso de enseñanza-aprendizaje-evaluación ${ }^{4}$. Por estas razones desde el año 2010, la Facultad de Medicina de la Universidad Finis Terrae ha implementado un programa de perfeccionamiento denominado "Diplomado en Pedagogía Universitaria en Ciencias de la Salud", cuyo objetivo es capacitar en prácticas educacionales efectivas a profesionales de la salud que ejercen docencia en la Facultad.

El objetivo del estudio es evaluar el impacto del Programa de capacitación docente sobre áreas de planificación de programas de curso, conducción de clases presenciales y rendimiento académico de los estudiantes.

\section{Material y Método}

El presente estudio retrospectivo, evaluó el resultado del Diplomado en Pedagogía Universitaria sobre: planificación de programas de curso, conducción de clases presenciales y mejoría en el rendimiento académico de los estudiantes. Para ello se analizaron los desempeños docentes antes del perfeccionamiento (2011) y después del perfeccionamiento (2012).

El diseño cuasi-experimental se describe en la Figura 1.

Se consideró como población de estudio a todos los docentes encargados de una o más asignaturas (académicos que asumen la responsabilidad de impartir totalmente una asignatura contenida en el plan de estudios) de las carreras de Kinesiología, Enfermería, Nutrición y Medicina y que la hubiesen impartido durante los años 2011 y 2012 en la Universidad.

Se excluyeron del estudio:

I. Profesores que efectuaron docencia durante el 2011 y estuvieron ausentes en el 2012.

II. Profesores cuya asignatura cambió de semestre durante el 2011 y 2012 y por tanto existió cambios en los conocimientos previos de los estudiantes.

III. Profesores encargados de asignatura cuyo ramo sufrió modificaciones en contenido o en su carga académica de un año a otro.

De un universo de 462 docentes pertenecientes a alguna de las 4 carreras de la Facultad, 324 fueron excluidos por ser docentes clínicos y/o docentes ayudantes de asignaturas. Sólo 138 docentes encargados de asignaturas cumplieron los requisitos de inclusión. De estos, 36 docentes fueron excluidos: 30 docentes por criterio I, 3 docentes por criterio II y 3 docentes por criterio III. La muestra quedó conformada finalmente por un total de 102 docentes, de los cuales 55 participaron del programa de perfeccionamiento (Tablas 1 y 2 ).

El diplomado se diseñó en 5 módulos semi-presenciales y abordó las temáticas deficitarias detectadas en un estudio realizado en la Facultad de Medicina el año 2010 (Figura 2). Cada módulo consistió de clases presenciales ( 4 h cada 15 días),

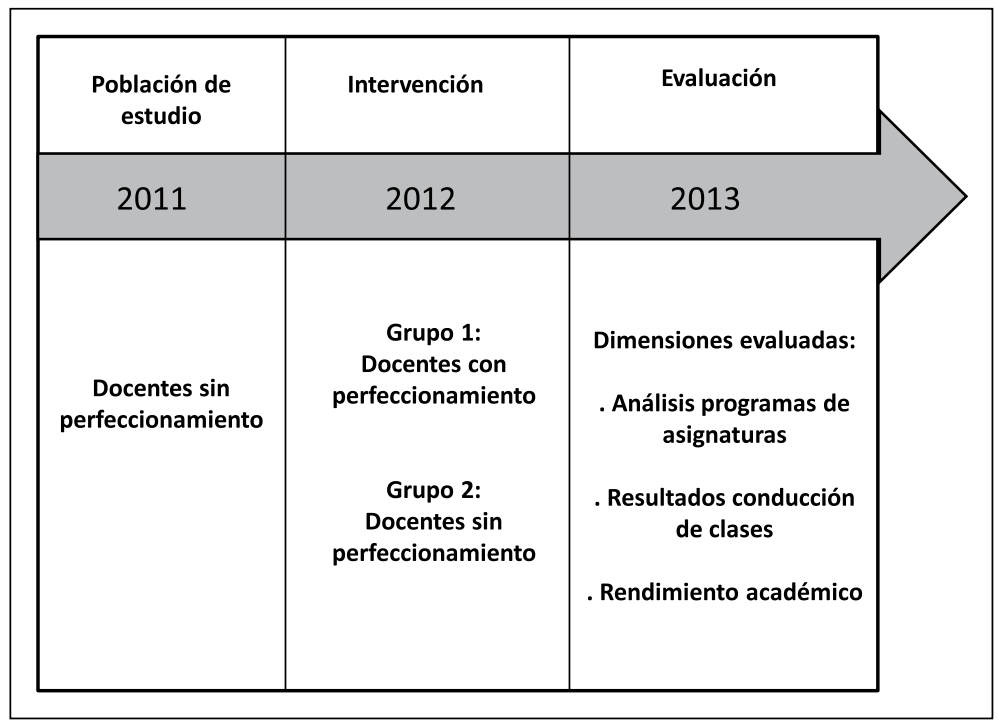

Figura 1. Diseño del estudio. 
Tabla 1. Distribución de docentes según escuela pertenecientes a la Facultad de Medicina

\begin{tabular}{|lccccc|}
\hline Escuelas & Medicina & Kinesiología & Nutrición & Enfermería & Total \\
\hline Total docentes & 217 & $9 G$ & 96 & 53 & 462 \\
\hline Tutores clínicos y/o ayudantes & 177 & 66 & 58 & 23 & 324 \\
\hline Encargados de asignaturas & 40 & 30 & 38 & 30 & 138 \\
\hline
\end{tabular}

Tabla 2. Distribución de la muestra

\begin{tabular}{|lcccc|}
\hline Universo muestral & Universo & $\begin{array}{c}\text { Cumplen con criterios } \\
\text { de inclusión }\end{array}$ & $\begin{array}{c}\text { Se excluyen } \\
\text { del estudio }\end{array}$ & Total muestra \\
\hline Docentes Facultad & 462 & 138 & 36 & 102 \\
\hline Medicina & 217 & 40 & 14 & 26 \\
\hline Kinesiología & 96 & 30 & 10 & 20 \\
\hline Nutrición & 96 & 38 & 9 & 29 \\
\hline Enfermería & 53 & 30 & 3 & 27 \\
\hline
\end{tabular}

Tabla 3. Criterios de cumplimiento y tipo de instrumento aplicado para la recogida de información del estudio

\begin{tabular}{|llcc|}
\hline Criterios de cumplimiento & Instrumento aplicado & No criterios & No indicadores \\
$\begin{array}{l}\text { 1. Calidad de Planificación docente mediante } \\
\text { análisis de programas de asignaturas }\end{array}$ & Pauta de cotejo & 7 & 21 \\
$\begin{array}{l}\text { 2. Calidad de Conducción de clases mediante } \\
\text { observación de clases }\end{array}$ & $\begin{array}{l}\text { Pauta de cotejo y encuesta } \\
\text { estudiantil }\end{array}$ & 3 & 13 \\
$\begin{array}{l}\text { 3. Rendimiento logrado mediante resultados } \\
\text { finales obtenidos en cada asignatura }\end{array}$ & $\begin{array}{l}\text { Registro calificaciones logradas } \\
\text { al finalizar los cursos }\end{array}$ & $\begin{array}{l}\text { Aprobaciones/ } \\
\text { Reprobaciones }\end{array}$ & $<4,00>4,0$ \\
\hline
\end{tabular}

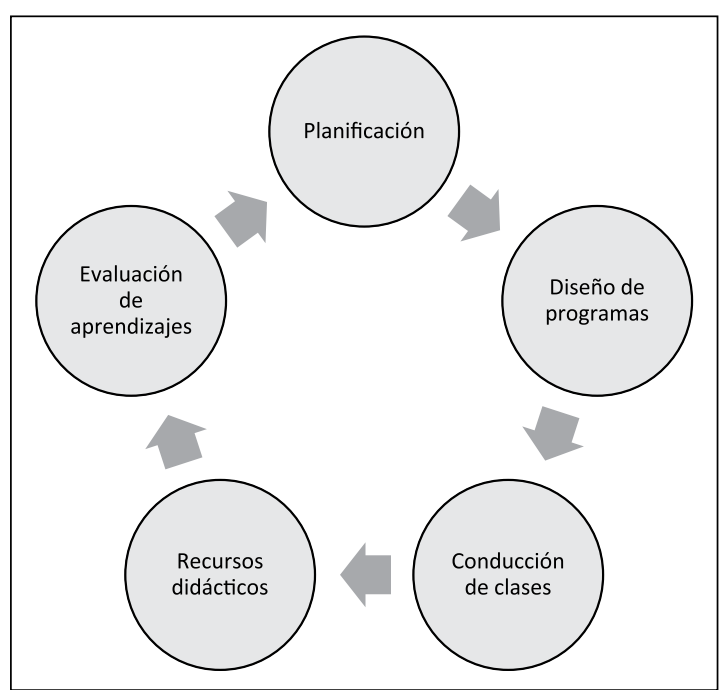

Figura 2. Temáticas abordadas en el Diplomado en Pedagogía universitaria según necesidades docentes detectadas. seguidas de actividades no presenciales de aplicación de contenidos, recibiendo retroalimentación permanente vía web. La duración del programa fue de $100 \mathrm{~h}$ cronológicas (50\% presenciales).

Para determinar el efecto del Diplomado en la docencia impartida, se establecieron los siguientes criterios de evaluación (Tabla 3):

\section{Calidad de la planificación docente}

Realizada mediante análisis de programas de asignaturas a través de una pauta de evaluación la cual definió 7 criterios de análisis y 21 indicadores. Estos criterios derivaron de un exhaustivo análisis de la literatura ${ }^{2,5,6}$ y de los estándares fijados por la Universidad Finis Terrae y su Facultad de Medicina (Tabla 4).

\section{Calidad de la conducción de clases}

Realizada mediante la aplicación de una pauta 
Tabla 4. Porcentaje de cumplimiento de indicadores según criterios de calidad para la elaboración de programas de curso

\begin{tabular}{|c|c|c|c|c|c|}
\hline Criterios & Indicadores & $\begin{array}{l}\text { Docentes } \\
\text { perfeccio- } \\
\text { nados }\end{array}$ & $\begin{array}{l}\text { Docentes } \\
\text { no perfec- } \\
\text { cionados }\end{array}$ & $\begin{array}{l}\text { Valor } p \\
\text { (por } \\
\text { indicador) }\end{array}$ & $\begin{array}{l}\text { Valor } p \\
\text { (por } \\
\text { criterio) }\end{array}$ \\
\hline \multirow{3}{*}{$\begin{array}{l}\text { Descripción } \\
\text { del curso }\end{array}$} & Se evidencia los propósitos del curso & $100 \%$ & $96 \%$ & 0,2099 & \multirow{3}{*}{$<0,0001^{* * *}$} \\
\hline & $\begin{array}{l}\text { Da cuenta en fama general de la metodología } \\
\text { del curso }\end{array}$ & $95 \%$ & $74 \%$ & $0,0052^{\prime \prime}$ & \\
\hline & $\begin{array}{l}\text { Señala quienes son los destratados y que se espera } \\
\text { de ellos }\end{array}$ & $100 \%$ & $70 \%$ & $<0,0001^{* * *}$ & \\
\hline \multirow{4}{*}{$\begin{array}{l}\text { Objetivos } \\
\text { específicos }\end{array}$} & \multirow{4}{*}{$\begin{array}{l}\text { Presenta conducta observable } \\
\text { El contenido es preciso } \\
\text { El contenido se desprende del objetivo general } \\
\text { Reflejan los contenidos procedimentales y/o actitu- } \\
\text { dinales involucrado en el objetivo general }\end{array}$} & $100 \%$ & $30 \%$ & $<0,0001^{* * *}$ & \multirow{4}{*}{$<0,0001^{* * *}$} \\
\hline & & $100 \%$ & $70 \%$ & $<0,0001 * * *$ & \\
\hline & & $100 \%$ & $17 \%$ & $<0,0001 * * *$ & \\
\hline & & $100 \%$ & $46 \%$ & $<0,0001 * * *$ & \\
\hline \multirow{5}{*}{$\begin{array}{l}\text { Formulación } \\
\text { de la compe- } \\
\text { tencia a lograr }\end{array}$} & Se formula la competencia considerando el saber & $100 \%$ & $30 \%$ & $<0,0001^{* * *}$ & \multirow{5}{*}{$<0,0001 * * *$} \\
\hline & $\begin{array}{l}\text { Se formula la competencia considerando el saber } \\
\text { hacer }\end{array}$ & $90 \%$ & $30 \%$ & $<0,0001 * * *$ & \\
\hline & Se formula la competencia considerando el Ser & $100 \%$ & $30 \%$ & $<0,0001^{* * *}$ & \\
\hline & $\begin{array}{l}\text { Se formula la competencia considerando el } \\
\text { contexto }\end{array}$ & $80 \%$ & $0 \%$ & $<0,0001^{* * *}$ & \\
\hline & $\begin{array}{l}\text { La competencia es consistente con el o los objeti- } \\
\text { vo (s) específicos }\end{array}$ & $90 \%$ & $20 \%$ & $<0,0001^{* * *}$ & \\
\hline \multirow[t]{5}{*}{$\begin{array}{l}\text { Unidades y } \\
\text { contenidos }\end{array}$} & $\begin{array}{l}\text { Presenta las unidades o ejes temáticos principales } \\
\text { de la asignatura }\end{array}$ & $100 \%$ & $96 \%$ & 0,2099 & \multirow{5}{*}{$<0,0001 * * *$} \\
\hline & $\begin{array}{l}\text { Organiza los contenidos declarativos en función } \\
\text { de los ejes temáticos }\end{array}$ & $100 \%$ & $80 \%$ & $<0,0001^{* * *}$ & \\
\hline & $\begin{array}{l}\text { Organiza las destrezas o contenidos procedimen- } \\
\text { tales en función de los ejes temáticos }\end{array}$ & $100 \%$ & $40 \%$ & $<0,0001^{* * *}$ & \\
\hline & Se observan contenidos actitudinales & $80 \%$ & $0 \%$ & $<0,0001 * * *$ & \\
\hline & $\begin{array}{l}\text { Los contenidos planteados se sitúan al nivel co- } \\
\text { rrespondiente de su plan de estudios }\end{array}$ & $100 \%$ & $90 \%$ & 0,0184 & \\
\hline \multirow[t]{4}{*}{ Metodología } & $\begin{array}{l}\text { La estrategia metodológica es coherente con los } \\
\text { objetivos especias planteados }\end{array}$ & $100 \%$ & $20 \%$ & $<0,0001 * * *$ & \multirow{4}{*}{$<0,0001 * * *$} \\
\hline & $\begin{array}{l}\text { La estrategia metodológica presenta como prota- } \\
\text { gonista al estudiante }\end{array}$ & $100 \%$ & $50 \%$ & $<0,0001 * * *$ & \\
\hline & Articula la utilización de diversas técnicas & $100 \%$ & $20 \%$ & $<0,0001^{* * *}$ & \\
\hline & $\begin{array}{l}\text { Hace explícito la forma de lograr el contenido } \\
\text { procedimental y/o actitudinal }\end{array}$ & $75 \%$ & $0 \%$ & $<0,0001^{* * *}$ & \\
\hline \multirow[t]{3}{*}{ Evaluación } & $\begin{array}{l}\text { Describe un sistema de evaluación consistente con } \\
\text { los objetivos específicos }\end{array}$ & $100 \%$ & $20 \%$ & $<0,0001 * * *$ & \multirow{3}{*}{$<0,0001 * * *$} \\
\hline & $\begin{array}{l}\text { Explicita instrumentos de evaluación que midan } \\
\text { contenidos declarativos }\end{array}$ & $100 \%$ & $98 \%$ & 0,4608 & \\
\hline & $\begin{array}{l}\text { Explicita instrumentos de evaluación que midan } \\
\text { contenidos procedimentales y/o actitudinales }\end{array}$ & $70 \%$ & $20 \%$ & $<0,0001^{* * *}$ & \\
\hline \multirow[t]{2}{*}{ Bibliografía } & $\begin{array}{l}\text { Considera referencias bibliográficas de los últimos } \\
5 \text { años }\end{array}$ & $100 \%$ & $98 \%$ & 0,4608 & \multirow[b]{2}{*}{0,2111} \\
\hline & $\begin{array}{l}\text { Las referencias bibliográficas están planteadas de } \\
\text { acuerdo a las normas establecidas por convenios } \\
\text { internacionales (APA; Vancouver u otra) }\end{array}$ & $100 \%$ & $96 \%$ & 0,2099 & \\
\hline
\end{tabular}


de cotejo especialmente diseñada (validada por criterio de jueces) y que consideró tres criterios de evaluación: Inicio, Desarrollo y Cierre de una clase, con un total de 13 indicadores de logro, demostrados útiles por la literatura ${ }^{7-11}$. La pauta de observación de clases fue aplicada por dos tipos de evaluadores: a) evaluadores pares (LS y CW) obteniendo 144 registros evaluados y b) estudiantes que respondieron 1.524 encuestas. Considerando la dificultad práctica para la observación directa de clases, se seleccionó una muestra de profesores a ser evaluados, en función de los siguientes criterios:

- Profesores que realizan sólo clases presenciales (excluyendo talleres, seminarios, laboratorios y actividades grupales).

- Clases entre 08:30 y 11:30 am.

- Clases con el curso completo (se excluyen secciones).

Se evaluaron 36 profesores; 50\% participó y aprobó el programa de perfeccionamiento y $50 \%$ no lo realizó.

La pauta de cotejo se aplicó simultáneamente $y$ en forma independiente por: dos observadores (LS y CW) y por los estudiantes de los cursos observados, antes del perfeccionamiento de los profesores y después del mismo. La información recogida desde estas tres fuentes, permitió validar los resultados (Tabla 5).
La validez consecuencial del instrumento se determinó mediante aplicaciones consecutivas. La confiabilidad no psicométrica se trabajó resguardando la suficiente evidencia de los aprendizajes siguiendo los parámetros de Brookhart ${ }^{12}$.

\section{Rendimiento académico}

Con el fin de observar el impacto del perfeccionamiento docente sobre el rendimiento se analizó el promedio de las calificaciones finales obtenidas por los estudiantes en los 36 cursos estudiados y el porcentaje de aprobación vs reprobación en cada uno.

\section{Análisis estadístico}

El análisis de distribución muestral utilizó pruebas de Kolmogorov-Smirnov, D-Agostino y Shapiro-Wilk mediante el programa GraphPad Prism para un $\alpha$ de 0,05 .

Para comparar los resultados de las tres áreas involucradas en el estudio, antes y después del perfeccionamiento se utilizó el t-test para muestras pareadas, y el test de Mann Whitney para muestras independientes.

Para evaluar el impacto de la capacitación docente sobre el rendimiento académico se aplicó el test exacto de Fisher y el cálculo de probabilidad OR (odds ratio).

Tabla 5. T-Student: Resultados comparativos entre docentes perfeccionados vs no perfeccionados antes y después de la intervención

\begin{tabular}{|c|c|c|c|c|c|c|c|}
\hline \multirow[t]{2}{*}{$\begin{array}{l}\text { Criterios de } \\
\text { conducción }\end{array}$} & \multirow[t]{2}{*}{ Resumen indicadores } & \multicolumn{3}{|c|}{$\begin{array}{l}\text { \% de logro al inicio del } \\
\text { semestre académico }\end{array}$} & \multicolumn{3}{|c|}{$\begin{array}{l}\text { \% de logro al final el } \\
\text { semestre académico }\end{array}$} \\
\hline & & Grupo 1 & Grupo 2 & Valor p & Grupo 1 & Grupo 2 & Valor $\mathbf{p}$ \\
\hline \multirow[t]{3}{*}{ Inicio } & Señala objetivos & 66 & $5 S$ & 0,1825 & 94 & 50 & $<0,0001$ \\
\hline & Propone desafío & 56 & 50 & 0,4698 & 70 & 50 & 0,0236 \\
\hline & Indica finalidad & 75 & 70 & 0,4499 & 80 & 65 & $<0,0001$ \\
\hline \multirow[t]{7}{*}{ Desarrollo } & Panorama de actividades & 61 & 60 & 0,7987 & 92 & 60 & $<0,0001$ \\
\hline & Entrega instrucciones & 77 & 72 & 0,4046 & 94 & 73 & 0,0044 \\
\hline & Atiende consultas & 85 & 74 & 0,1498 & 96 & 76 & 0,0101 \\
\hline & Entrega los tiempos & 76 & 74 & 0,8474 & 88 & 76 & 0,1823 \\
\hline & Promueve trabajo colaborativo & 67 & $5 S$ & 0,0907 & 85 & 56 & 0,0007 \\
\hline & Relaciona actividades & 68 & 63 & 0,2139 & 90 & 55 & $<0,0001$ \\
\hline & Las actividades ordena ideas & 80 & 63 & 0,1653 & 90 & 65 & $<0,0001$ \\
\hline \multirow[t]{3}{*}{ Cierre } & Actividades de síntesis & 62 & 55 & 0,2968 & 93 & 50 & $<0,0001$ \\
\hline & Puesta en común & 54 & 49 & 0,2993 & 74 & 44 & $<0,0001$ \\
\hline & Da tiempos para síntesis & 53 & 57 & 0,1808 & 81 & 49 & $<0,0001$ \\
\hline
\end{tabular}




\section{Resultados}

\section{Calidad de la planificación docente mediante análisis de programas de asignaturas}

Se revisaron 102 programas de asignaturas, 55 correspondientes a profesores perfeccionados y 47 a no perfeccionados. El promedio de cumplimiento de los criterios de calidad analizados se muestran en la Figura 3.

En 6 de los 7 criterios de calidad considerados, el porcentaje de cumplimiento es significativamente mayor en los profesores perfeccionados. $(\mathrm{p}<0,0001)$ al igual que en 21 de los
26 indicadores estudiados. Sólo en 5 no hubo diferencias significativas, debido al alto grado de cumplimiento alcanzado por ambos grupos $(\geq 96 \%)$ (Tabla 4).

\section{Calidad de la Conducción de clases}

La influencia del programa de perfeccionamiento sobre calidad de conducción de clases, en los 36 docentes estudiados (al inicio y fin del semestre) respecto del Inicio, Desarrollo y Cierre de una clase, se observan en la Figura 4. Si bien las formas de conducción de clases de ambos grupos no presentan diferencias significativas durante la

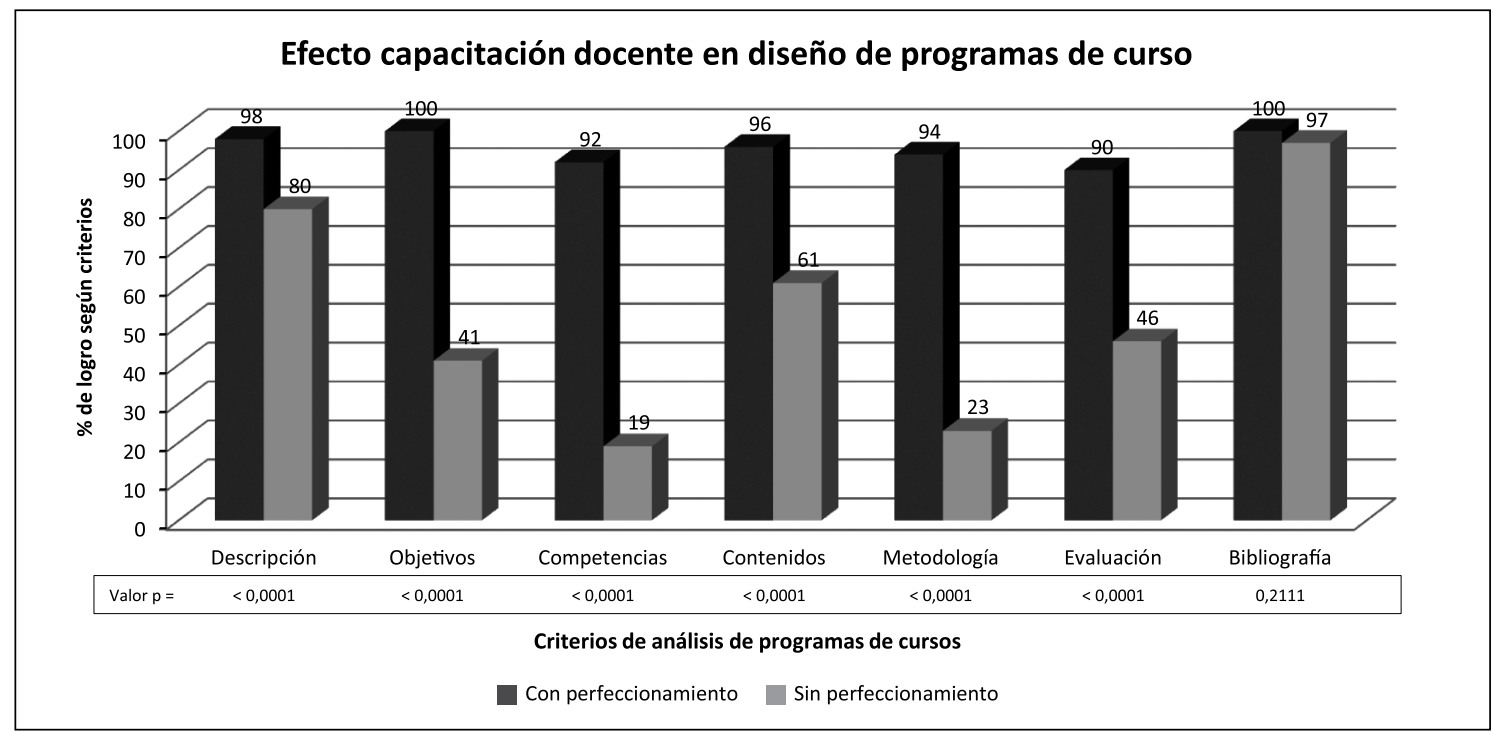

Figura 3. Promedio de los porcentajes de cumplimiento de criterios entre docentes con y sin perfeccionamiento en el diseño de programas de asignaturas.

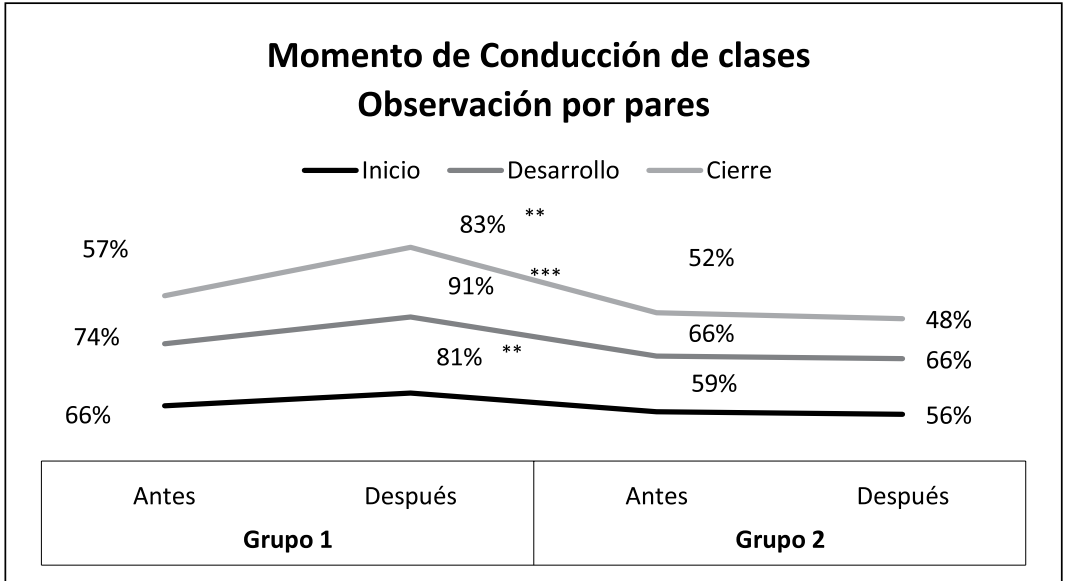

Figura 4. Promedio de los porcentajes según criterios de logro en función de los momentos de conducción de las clases. Grupo de docentes antes y después del perfeccionamiento. 
evaluación inicial, el grupo de docentes perfeccionados mejoran significativamente la evaluación por pares, tanto en las fases de inicio, desarrollo como cierre de una clase luego de su capacitación.
Esta diferencia no se observa en el grupo de docentes no perfeccionados quienes mantienen sin variación la calificación de su actividad al inicio y fin del semestre en estudio (Figura 5).

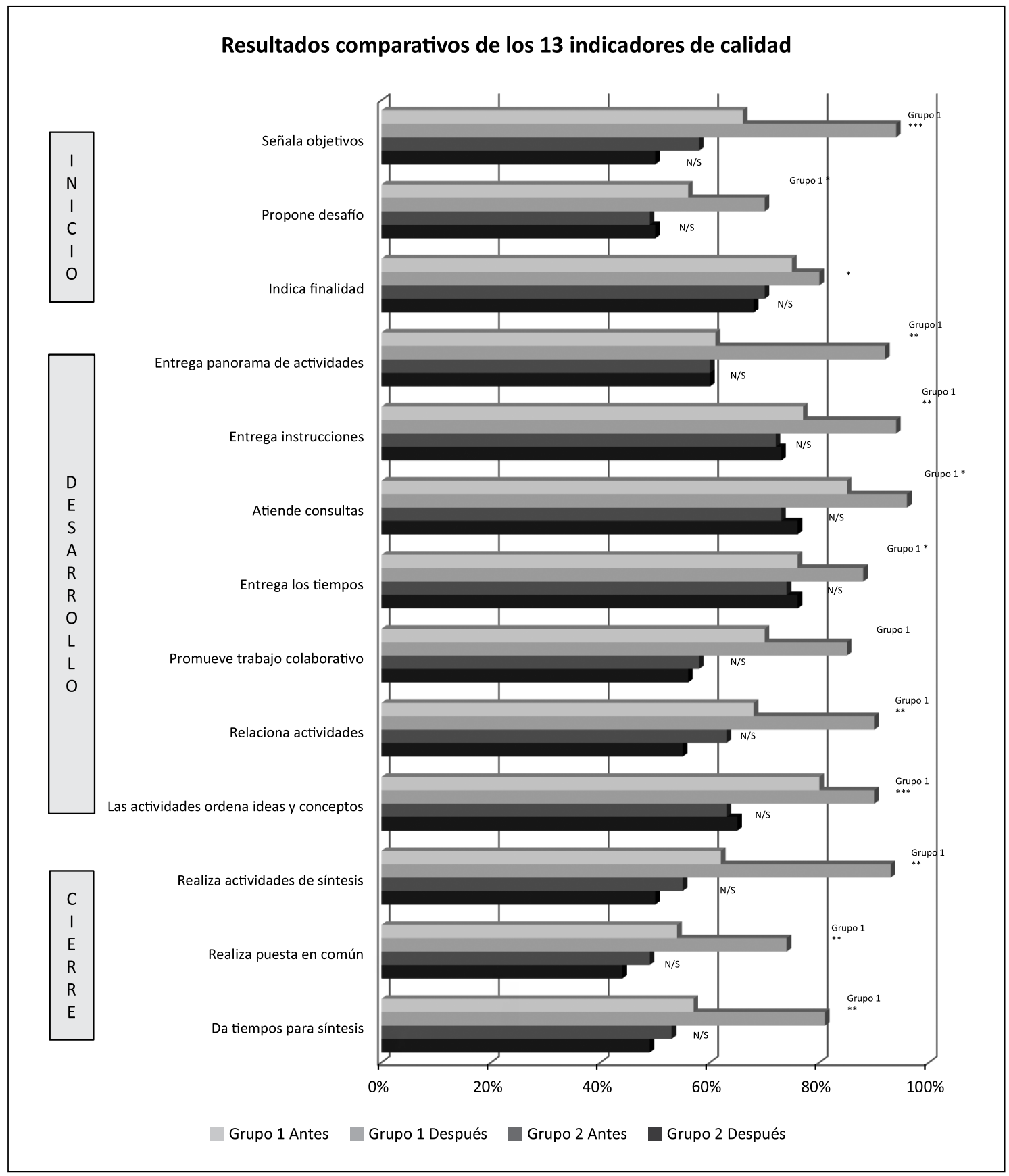

Figura 5. Comportamiento observado por pares evaluadores antes y después del perfeccionamiento respecto de los 13 indicadores de calidad de conducción de clases. 
Tabla 6. Resultados comparativos entre docentes perfeccionados vs no perfeccionados durante el semestre académico 2011 y 2012

\begin{tabular}{|c|c|c|c|}
\hline Grupos de docentes & 2011 & 2012 & Valor $\mathbf{p}$ \\
\hline G1: con perfeccionamiento & 4,49 & 5,24 & $<0,0001$ \\
\hline G2: sin perfeccionamiento & 4,44 & 4,51 & 0,4975 \\
\hline
\end{tabular}

Tabla 7. Test exacto de Fisher's; Resultados según tabla de contingencia entre estudiantes con profesor perfeccionado (Grupo 1) o no perfeccionado (Grupo 2) vs número de aprobaciones por asignatura

\begin{tabular}{|rrrr|}
\hline Grupo 1 & Aprueban & Reprueban & Total \\
\hline 2012 & 880 & 58 & 938 \\
\hline 2011 & 739 & 220 & 959 \\
\hline Total & 1.619 & 278 & 1.897 \\
\hline
\end{tabular}

$\mathrm{p}<0,0001$; Odds ratio: 4,5; 95\% Cl 3,328 a 6,130.

\begin{tabular}{|cccr|}
\hline Grupo 2 & Aprueban & Reprueban & Total \\
2012 & 660 & 299 & 959 \\
2011 & 702 & 265 & 967 \\
\hline Total & 1.362 & 564 & 1.926 \\
\hline
\end{tabular}

$\mathrm{p}<0,0715$; Odds ratio: 0,$8 ; 95 \% \mathrm{Cl} 0,6845$ a 1,014 .

Los resultados obtenidos por la evaluación efectuada por los alumnos asistentes a las clases, muestran iguales diferencias significativas a favor de los docentes que fueron perfeccionados (Tabla 6).

\section{Rendimiento académico}

Para medir el efecto del programa de perfeccionamiento sobre el rendimiento académico de los estudiantes, se revisaron 3.823 calificaciones finales obtenidas en los 36 cursos del estudio: 1.897 para el grupo 1 (G1: docentes perfeccionados) y 1.926 para el grupo 2 (G2: docentes no perfeccionados). Se compararon las notas obtenidas para ambos grupos durante los años 2011 y 2012 (antes y después del perfeccionamiento). Los resultados se muestran en la Tabla 7.

Los alumnos cuyos docentes fueron perfeccionados (G1:2012) elevan su rendimiento en forma significativa al compararlo con los alumnos que tuvieron al mismo docente previo al perfeccionamiento (G1: 2011). En contraste, los estudiantes del grupo de docentes no perfeccionados $(\mathrm{G} 2)$, no
Tabla 8. Test Mann-Whitney; Resultados comparativos entre calificaciones totales 2011 y calificaciones totales 2014

\begin{tabular}{|lcc|}
\hline \multicolumn{3}{|c|}{ Mann-Whitney test } \\
\hline Valores analizados & 7.040 & 12.243 \\
\hline Promedio & 4,922 & 5,389 \\
\hline Desviación estándar & 0,9854 & 0,7438 \\
Error estándar & 0,01174 & 0,006722 \\
\hline p valor & $<0,0001$ \\
\hline
\end{tabular}

muestran diferencias importantes en sus calificaciones en ambos períodos de estudio.

Las tasas de aprobación/reprobación difieren significativamente según la capacitación del profesor ( $<<0,0001$, OR: 4,5) (Tabla 7).

Dado que el número de docentes perfeccionado al año 2014 ha aumentado considerablemente $(\mathrm{n}=233)$, se comparó el rendimiento académico global de los alumnos entre los años 2011 y 2014. Se compararon 7.044 notas de 2011 y 12.243 notas de 2014, encontrando diferencias significativas en favor de este último $(\mathrm{p}<0,0001)$ (Tabla 8).

\section{Discusión}

Las escuelas de medicina han asumido históricamente que todo académico está capacitado para efectuar docencia en forma autodidacta. Sin embargo, para impartir una docencia de calidad que estimule un aprendizaje efectivo se requiere de formación docente. Si bien muchos autores dan cuenta de la relación existente entre desempeño docente y rendimiento académico, ninguno de ellos muestra en detalle los indicadores que sería necesario evaluar para reconocer un adecuado desempeño docente ${ }^{13-15}$ el cual no sólo se refiere a la transmisión de conocimiento ${ }^{4,13,14}$, sino 
que implica una planificación intencionada que repercute en los procesos cognitivos y afectivos de los estudiantes y sus aprendizajes ${ }^{13}$, los cuales repercuten -a su vez- en el rendimiento académico alcanzado $^{13-19}$.

Este estudio propone determinantes de desempeño específicos, con sus correspondientes indicadores de logro, los cuales debieran incorporarse a los programas de capacitación docente en ciencias de la salud, como guía que oriente su planificación y desarrollo, con el propósito de impactar positivamente en el aprendizaje de los estudiantes. Esta propuesta permite evaluar el desempeño académico y establecer su impacto directo en la docencia, tal como lo propone el cuarto nivel del modelo de Kirckpatric ${ }^{20}$ (primer nivel evalúa las variables de organización, contenidos, métodos y profesores que han dictado la capacitación; segundo nivel o aprendizaje, evalúa la adquisición de conocimientos y destrezas y/o el cambio en las percepciones hacia lo aprendido por parte de los participantes; el tercer nivel o conducta, evalúa la disposición de los participantes para aplicar conocimientos y destrezas y/o practicar lo aprendido en su lugar de trabajo; cuarto nivel o resultados, mide impacto sobre rendimiento académico) lo cual no ha sido evaluado previamen$\mathrm{te}^{21,22}$. Nuestros resultados avalan la importancia del perfeccionamiento académico para mejorar resultados en el proceso enseñanza-aprendizaje. Queda en evidencia que los docentes mejoraron significativamente en diseño de objetivos y competencias, selección de contenidos, metodologías de enseñanza y evaluación lo cual incidió positivamente en la conducción de clases y rendimiento de sus estudiantes. Al generar un clima inicial motivante y señalar los objetivos y actividades a desarrollar durante la clase, los estudiantes evocan sus conocimientos previos y activan sus esquemas de pensamiento relacionados con la materia a desarrollar. Mediante las actividades propuestas durante el desarrollo de la misma, los estudiantes organizan sus ideas y junto a la orientación del docente, destinan tiempos para sintetizar sus conocimientos, sellar sus aprendizajes almacenarlos de una manera ordenada y sistemática que facilita su evocación y transferencia. Este trabajo permite efectuar mejores instrumentos de evaluación que reflejen más adecuadamente los aprendizajes reales alcanzados.

$\mathrm{Al}$ año 2014, es posible señalar que los resul- tados del aprendizaje de nuestros estudiantes continúan mejorando como consecuencia de la continuidad del programa de perfeccionamiento docente y de la difusión de buenas prácticas.

En base a la información obtenida, se puede sugerir que la capacitación docente en las áreas de ciencias de la salud se podría realizar mediante programas que entreguen una formación pedagógica orientada en base a indicadores precisos y relacionados con el diseño y planificación de programas de cursos, de clases y de su conducción ${ }^{21,23}$.

Esta propuesta de programa de capacitación tiene validez en el contexto de clases lectivas de cualquier nivel de la enseñanza en ciencias de la salud, sin embargo, no ha sido diseñada para ser aplicada en tutorías clínicas, las cuales requieren adicionar otros criterios y metodologías docentes ${ }^{24,25}$.

\section{Referencias}

1. López F. Tendencias de la Educación Superior en el mundo y en América Latina y el Caribe. Avaliação, Campinas; Sorocaba 2008; 13: 267-91.

2. Hiebert J, Morris A, Berk D, Jansen A. Preparing teachers to learn from teaching. Journal of Teacher Education 2007; 58: 47-61.

3. Lindstrom J. A System for Quality Improvement in Higher Education. European. Journal of Engineering Education 1994; 19: 255-61.

4. Alterterio G, Pérez H. Evaluación de la función docente según desempeño de los profesores y la opinión estudiantil. Revista Educación Médica Superior 2009; 23: 1-14.

5. Zabalza MA. Competencias docentes del profesorado universitario. Madrid: Narcea, 2003.

6. Wheatley KF. The potential benefits of teacher efficacy doubts for educational reform. Teaching and Teacher Education 2002; 18: 5-22.

7. De la Orden A, Muñoz R, Fernández MJ, Fuentes A, García JM, Guardia S, et al. Desarrollo y Validación de un Modelo de Calidad Universitaria como base para su Evaluación. Revista Electrónica de Investigación y Evaluación Educativa 1997; 3. Número 1_2. En http://www. uv.es/relieve/v3n1/RELIEVEv3n1_2.htm. (Consultado el 20 julio de 2014).

8. Carrasco R, Torrecilla F J. A avaliação das aprendizagens na América Latina. Comportamentos e tendências do desempenho dos estudantes latinoamericanos nos ensinos primários e secundários. Sísifo. Revista de Ciência da Educação, 2009; 09: 31-46. 
9. Coll C, Martín E, Mauti T, Miras Y, Onrubia J, Solé I, et al. El constructivismo en el aula. 18ava edición, Editorial GRAÖ: Barcelona. 2007.

10. Montero L, Triviño X, Sirhan M, Moore P, Leiva L. Barreras para la formación en docencia de los profesores de medicina: una aproximación cualitativa. Santiago, Chile: Rev Med Chile 2012; 140: 695-702.

11. Mc Millan JH. Understanding and improving teacher`s classroom assessment decision making: Implications for Theory and Practice. Educational measurement Issues and Practice 2003; 22 (4): 34-43.

12. Brookhart S. Developing measurement theory for classroom assessment. Purposes and uses Educational measurement: Issues and Practice 2003; 22 (4): 5-12.

13. Montero E, Villalobos J, Valverde A. Factores Institucionales, Pedagógicos, Psicosociales y Sociodemográficos asociados al Rendimiento Académico en la Universidad de Costa Rica: Un Estudio multinivel. Relieve 2007; 13: 215-34.

14. Page M, Moreal B, Calleja J, Cerdan J, Echeverría M, García C, et al. Hacia un modelo causal del rendimiento académico. Madrid, España: Centro de publicaciones del Ministerio de Educación y Ciencia.(CIDE).1990.

15. Santos M. Evaluación Educativa. Tomos I y II. Buenos Aires: Editorial Magisterio del Río de la Plata; 1996.

16. Ellis W. Quality assurance for university teaching. London: Society for research in higher education; 1993.

17. Gibson S, Dembo M. Teacher`s Sense of Efficacy: An
Important Factor in School Improvement. The Elementary School Journal 1985; 86: 173-84.

18. Huertas JA. Motivación: Querer Aprender. Buenos Aires: Aique. 1997.

19. Pérez Luño A, Ramón J, Sánchez J. Análisis exploratorio de las variables que condicionan el rendimiento académico. Sevilla, España: Universidad Pablo de Olavide. 2000.

20. Kirckpatric D. Evaluación de acciones formativas: los 4 niveles. Barcelona, España: Editorial Gestión 2000; 1999.

21. McLean M, Cilliers F, Van Wyk JM. Faculty development: yesterday, today and tomorrow. Med Teach 2008; 30: 555-84.

22. Sirhan M, Triviño X. Evaluación de una experiencia de capacitación en planificación educacional para directores de programas de las especialidades médicas. Santiago, Chile: Rev Med Chile 2012; 140: 530-7.

23. Santelices L, Williams C. Pedagogía en Ciencias de la Salud: Teoría y Praxis para una enseñanza universitaria. Santiago, Chile: Ediciones Universidad Finis Terrae; 2013.

24. Paice E, Heard S, Moss F. How important are role models in making good doctors?. British Medical Journal 2002; 325: 707-10.

25. Vélez Van M, Roa NC. Factors associated with academic perfomance in medical students. En: PSIC. Educación Médica 2005; 2: 1-10. 